\title{
The Structure of Simple Modules of Birman-Murakami-Wenzl Algebras
}

\begin{abstract}
$\mathbf{X u ~ X u}$
School of Mathematics and Statistics, Wuhan University, Wuhan 430072, China

Correspondence should be addressed to Xu Xu; xuxu2013@whu.edu.cn

Received 6 July 2015; Revised 16 September 2015; Accepted 20 September 2015

Academic Editor: Naihuan Jing

Copyright (C) $2015 \mathrm{Xu} \mathrm{Xu}$. This is an open access article distributed under the Creative Commons Attribution License, which permits unrestricted use, distribution, and reproduction in any medium, provided the original work is properly cited.

We study the restriction of simple modules $D^{f, \lambda}$ of Birman-Murakami-Wenzl algebras $\mathscr{B}_{n}(r, q)$ with $q$ being not a root of 1 . Precisely, we study the module structure for the restriction of $D^{f, \lambda}$ to $\mathscr{B}_{n-1}(r, q)$ and describe the socle and head of the restriction of each simple module completely.
\end{abstract}

\section{Introduction}

Classical Schur-Weyl duality relates the representations of the symmetric and general linear groups via their actions on tensor space. Brauer [1] introduced a class of algebras called Brauer algebras to generalize the classical Schur-Weyl duality. He proved that there is a Schur-Weyl duality between Brauer algebras with some special parameters over $\mathbb{C}$ and orthogonal or symplectic groups.

Birman-Wenzl [2] and Murakami [3] introduced a class of associative algebras $\mathscr{B}_{n}(r, q)$ independently, called BirmanMurakami-Wenzl algebras, in order to study link invariants. On the other hand, there is a Schur-Weyl duality between $\mathscr{B}_{n}(r, q)$ with some special parameters over $\mathbb{C}$ and quantum groups of types B, C, and D [4]. So, BMW algebras can be seen as the $q$-deformation of Brauer algebras.

Recently, based on the results of decomposition numbers of Brauer algebras, De Visscher and Martin [5] described the module structure of the restriction of simple modules of Brauer algebras by using certain combinatorial graph. In [6], the author gave a combinatorial algorithm for computing decomposition numbers of BMW algebras. Motivated by these works, we study the restriction of simple modules of BMW algebras in this paper.

We organize this paper as follows: in Section 2, we recall some results on representation theory of BMW algebras. Then, we will describe the structure of the restriction from $\mathscr{B}_{n}(r, q)$ to $\mathscr{B}_{n-1}(r, q)$ for simple modules in Section 3.

\section{Birman-Murakami-Wenzl Algebra}

In this section, we recall some results on the BMW algebra $\mathscr{B}_{n}(r, q)$ [2] over an integral domain $R:=\mathbb{Z}\left[r^{ \pm 1}, q^{ \pm 1}, \omega^{-1}\right]$, where $\omega=q-q^{-1}$ and $q, r$ are indeterminates.

Definition 1 (see [2]). The BMW algebra $\mathscr{B}_{n}(r, q)$ is a unital associative $R$-algebra generated by $T_{i}, 1 \leq i<n$ subject to the following relations:

(a) $\left(T_{i}-q\right)\left(T_{i}+q^{-1}\right)\left(T_{i}-r^{-1}\right)=0$, for $1 \leq i<n$,

(b) (1) $T_{i} T_{j}=T_{j} T_{i}$ if $|i-j|>1$,

(2) $T_{i} T_{i+1} T_{i}=T_{i+1} T_{i} T_{i+1}$, for $1 \leq i<n-1$,

(c) (1) $E_{i} T_{i}=r^{-1} E_{i}=T_{i} E_{i}$, for $1 \leq i \leq n-1$,

(2) $E_{i} T_{j}^{ \pm 1} E_{i}=r^{ \pm 1} E_{i}$, for $1 \leq i \leq n-1$ and $j=i \pm 1$,

where $E_{i}=1-\omega^{-1}\left(T_{i}-T_{i}^{-1}\right)$ for $1 \leq i \leq n-1$.

It is well-known that the Hecke algebra $\mathscr{H}_{n}$ associated with the symmetric group $\delta_{n}$ is a quotient algebra of BMW algebra $\mathscr{B}_{n}(r, q)$. Morton and Wassermann [7] proved that $\mathscr{B}_{n}(r, q)$ is free over $R$ with rank $(2 n-1) ! !$.

When $\mathscr{B}_{n}(r, q)$ is semisimple, the simple modules are cell modules in the sense of [8]. The branching rule of cell modules is well-known [9]. However, the algebra $\mathscr{B}_{n}(r, q)$ is not always semisimple. In 2009, Rui and Si gave a necessary 
and sufficient condition for BMW algebras being semisimple over an arbitrary field [10].

It is different from Hecke algebra $\mathscr{H}_{n}$; BMW algebra $\mathscr{B}_{n}(r, q)$ may not be semisimple even if $q$ is not a root of 1. In this paper, we study the restriction of simple modules of BMW algebra $\mathscr{B}_{n}(r, q)$ over a field and with the assumption that $q$ is not a root of 1 . According to [10], we will assume $r=\varepsilon q^{a}$ for some $a \in \mathbb{Z}$ and $\varepsilon= \pm 1$. Otherwise, $\mathscr{B}_{n}(r, q)$ is semisimple.

Now, we need some of combinatorics in order to state the results on $\mathscr{B}_{n}(r, q)$.

Let $\Lambda_{n}=\left\{(f, \lambda) \mid 0 \leq f \leq\lfloor n / 2\rfloor, \lambda \in \Lambda^{+}(n-2 f)\right\}$, where $\Lambda^{+}(n-2 f)$ is the set of partitions of $n-2 f$. By definition, each $\lambda=\left(\lambda_{1}, \lambda_{2}, \ldots\right) \in \Lambda^{+}(n-2 f)$ is a weakly decreasing sequence of nonnegative integers such that $|\lambda|$, the summation of those integers, is $n-2 f$. For $(f, \lambda),(\ell, \mu) \in \Lambda_{n}$, we say that $(f, \lambda)$ dominates $(\ell, \mu)$ and write $(f, \lambda) \geq(\ell, \mu)$, if $f>\ell$ in the usual sense or $f=\ell$ and $\lambda \unrhd \mu$ in the sense that

$$
\sum_{k=1}^{l} \lambda_{k} \geq \sum_{k=1}^{l} \mu_{k}
$$

for all $l \geq 1$. So, $\Lambda_{n}$ is a poset. We write $\lambda \supseteq \mu$ if $\lambda_{j} \geq \mu_{j}$ for all possible $j$. Let $\Lambda=\bigcup_{n=1}^{\infty} \Lambda_{n}$ and $\Lambda^{+}=\bigcup_{n=1}^{\infty} \Lambda^{+}(n)$.

The Young diagram $Y(\lambda)$ for a partition $\lambda=\left(\lambda_{1}, \lambda_{2}, \ldots\right)$ is a collection of boxes with $\lambda_{i}$ boxes in the $i$ th row of $Y(\lambda)$. A box of $Y(\lambda)$ is called removable (resp., addable) if it can be removed from (resp., added to) $Y(\lambda)$ such that the result is still a Young diagram for a partition. A $\lambda$-tableau $\mathbf{s}$ is obtained by inserting $i, 1 \leq i \leq n$, into $Y(\lambda)$ without repetition. We denote the set of all removable boxes of $Y(\lambda)$ by $\mathscr{R}(\lambda)$ and the set of all addable boxes of $Y(\lambda)$ by $\mathscr{A}(\lambda)$.

We recall the definition of a cellular algebra in [8].

Definition 2 (see [8]). Let $R$ be a commutative ring and $A$ an $R$-algebra. Fix a partially ordered set $\Lambda=(\Lambda, \unrhd)$. Then for each $\lambda \in \Lambda$, let $T(\lambda)$ be a finite set and $C_{\text {st }}^{\lambda} \in A$, where $\mathbf{s}, \mathbf{t} \in$ $T(\lambda)$. The triple $(\Lambda, T, C)$ is a cell datum for $A$ if

(a) $\left\{C_{\text {st }}^{\lambda} \mid \lambda \in \Lambda\right.$ and $\left.\mathbf{s}, \mathbf{t} \in T(\lambda)\right\}$ is an $R$-basis for $A$;

(b) there is an $R$-linear anti-involution $*$ on $A$ such that $\left(C_{\mathbf{s t}}^{\lambda}\right)^{*}=C_{\mathbf{t s}}^{\lambda}$, for all $\lambda \in \Lambda$ and all $\mathbf{s}, \mathbf{t} \in T(\lambda) ;$

(c) we let $A^{\triangleright \lambda}=R$-span $\left\{C_{\mathbf{u v}}^{\mu} \mid \mu \triangleright \lambda\right.$ and $\left.\mathbf{u}, \mathbf{v} \in T(\mu)\right\}$. For any $\lambda \in \Lambda$, s $\in T(\lambda)$, and $a \in A$ there exist scalars $r_{\text {tu }}(a) \in R$ such that

$$
C_{\mathbf{s t}}^{\lambda} a=\sum_{\mathbf{u} \in T(\lambda)} r_{\mathbf{t u}}(a) C_{\mathbf{s u}}^{\lambda}\left(\bmod A^{\triangleright \lambda}\right) .
$$

Algebra $A$ is a cellular algebra if it has a cell datum.

$\mathrm{Xi}$ [11] proved that $\mathscr{B}_{n}(r, q)$ is a cellular algebra over $R$ associated with the poset $\Lambda_{n}$.

We recall the representations of $\mathscr{B}_{n}(r, q)$ over a field as follows. For each $(f, \lambda) \in \Lambda_{n}$, there is a cell module $\Delta(f, \lambda)$ for $\mathscr{B}_{n}(r, q)$. On each $\Delta(f, \lambda)$, there is an invariant form, say $\phi_{f, \lambda}$. Let $\operatorname{Rad} \Delta(f, \lambda)$ be the radical of $\phi_{f, \lambda}$. Then the corresponding quotient module $\Delta(f, \lambda) / \operatorname{Rad} \Delta(f, \lambda)$ is either zero or absolutely irreducible. In the latter case, we write it as $D^{f, \lambda}$. Let $P(f, \lambda)$ be the projective cover of $D^{f, \lambda}$.

Let $\bmod -\mathscr{B}_{n}(r, q)$ be the category of right $\mathscr{B}_{n}(r, q)$ modules. We have embedding of the BMW algebras

$$
\mathscr{B}_{n-1}(r, q) \hookrightarrow \mathscr{B}_{n}(r, q) \hookrightarrow \mathscr{B}_{n+1}(r, q)
$$

So, we have corresponding induction functor $\operatorname{ind}_{n}: \bmod$ $\mathscr{B}_{n}(r, q) \rightarrow \bmod -\mathscr{B}_{n+1}(r, q)$ and restriction functor res ${ }_{n}:$ $\bmod -\mathscr{B}_{n}(r, q) \rightarrow \bmod -\mathscr{B}_{n-1}(r, q)$. Note that res $_{n}$ is exact functor and ind $\mathrm{d}_{n}$ is right exact functor.

By standard arguments in [12, Section 6], Rui and Si defined the exact functor $\mathscr{F}_{n}: \bmod -\mathscr{B}_{n}(r, q) \rightarrow$ $\bmod -\mathscr{B}_{n-2}(r, q)$ and right exact functor $\mathscr{G}_{n-2}$ : $\bmod -\mathscr{B}_{n-2}(r, q) \rightarrow \bmod -\mathscr{B}_{n}(r, q)$ in [13], which satisfy

$$
\begin{aligned}
\mathscr{F}_{n}(M) & =M E_{n-1}, \\
\mathscr{G}_{n-2}(N) & =N \bigotimes_{\mathscr{B}_{n-2}(r, q)} E_{n-1} \mathscr{B}_{n}(r, q)
\end{aligned}
$$

for all $M \in \bmod -\mathscr{B}_{n}(r, q)$ and $N \in \bmod -\mathscr{B}_{n-2}(r, q)$. For the simplification of notation, we will use $\mathscr{F}$ and $\mathscr{G}$ instead of $\mathscr{F}_{n}$ and $\mathscr{G}_{n}$, respectively.

The following results were proved by Rui and $\mathrm{Si}$ for cyclotomic BMW algebras [14] and we only need the special case here.

Lemma 3 (see [14, Lemma 5.1]). Suppose that $(f, \lambda) \in \Lambda_{n}$ and $(\ell, \mu) \in \Lambda_{n+2}$. We have
(1) $\mathscr{F} \mathscr{G}=1$,
(2) $\mathscr{G}(\Delta(f, \lambda))=\Delta(f+1, \lambda)$,
(3) $\mathscr{F}(\Delta(f, \lambda))=\Delta(f-1, \lambda)$,
(4) $\operatorname{Hom}_{\mathscr{B}_{n+2}(r, q)}(\mathscr{G}(\Delta(f, \lambda)), \Delta(\ell, \mu))$
$\operatorname{Hom}_{\mathscr{B}_{n}(r, q)}(\Delta(f, \lambda), \mathscr{F}(\Delta(\ell, \mu)))$ as vector spaces.

At the end of this section, we recall the branching rule for cell modules of $\mathscr{B}_{n}(r, q)$ over $R$.

Theorem 4 (see [9]). For each $(f, \lambda) \in \Lambda_{n}$, then $\Delta(f, \lambda)$ has a filtration $0=M_{0} \subseteq M_{1} \subseteq \cdots \subseteq M_{m}=\Delta(f, \lambda)$ of $\mathscr{B}_{n-1}(r, q)$ modules such that $M_{i} / M_{i-1} \cong \Delta(\ell, \mu)$, where $\mu$ ranges over all partitions obtained from $\lambda$ by either removing a removable node if $\ell=f$ or adding an addable node if $\ell=f-1$. Further, the multiplicity of $\Delta(\ell, \mu)$ in $\operatorname{res}_{n} \Delta(f, \lambda)$ is one. In particular, this result is true over an arbitrary field.

Notation. If $\Delta(\ell, \mu)$ appear in the section defined by the above theorem, we write $(\ell, \mu) \rightarrow(f, \lambda)$.

In [6], the author proved the following result for cyclotomic BMW algebra and so for BMW algebra.

Lemma 5. $\operatorname{res}_{n+2}(?) \circ \mathscr{G}_{n}(?)=\operatorname{ind}_{n}(?)$.

Remark 6. Theorem 4, together with Lemmas 3 and 5, implies that there is a result for $\operatorname{ind}_{n} \Delta(f, \lambda)$ similar to Theorem 4 . Hence we will use Theorem 4 for $\operatorname{ind}_{n} \Delta(f, \lambda)$ with no additional comments. 


\section{The Structure of Simple Modules of $\mathscr{B}_{n}(r, q)$}

In this section, we describe the module structure of the restriction of $D^{f, \lambda}$ to $\mathscr{B}_{n-1}(r, q)$ for $(f, \lambda) \in \Lambda_{n}$.

For each partition $\lambda=\left(\lambda_{1}, \lambda_{2}, \ldots\right)$ and each $a \in \mathbb{Z}$, define $\rho_{a}, e_{a}(\lambda) \in \mathbb{R}^{\mathbb{N}}$ by

(1) $\rho_{a}=(-(a+1) / 2,-(a+3) / 2, \ldots)$ if $r=q^{a}$,

(2) $\rho_{a}=((a-1) / 2,(a-3) / 2, \ldots)$ if $r=-q^{a}$,

(3) $e_{a}(\lambda)=\lambda^{t}+\rho_{a}$ if $r=q^{a}$,

(4) $e_{a}(\lambda)=\lambda+\rho_{a}$ if $r=-q^{a}$,

where $\lambda^{t}$ is the conjugate of $\lambda$.

It is easy to see that $e_{a}(\lambda)$ is a strictly decreasing sequence. Let $s\left(e_{a}(\lambda)\right.$ ) (or $s(\lambda)$ for brevity) be the number of pairs $\{p,-p\}$ in $e_{a}(\lambda)$.

Example 7. Assume $r=-q^{3}$ and $\lambda=(3,2,1)$; then $\rho_{a}=$ $(1,0,-1,-2, \ldots)$ and $e_{a}(\lambda)=(4,2,0,-2,-3,-4,-5, \ldots)$. The pairs $\{p,-p\}$ in $e_{a}(\lambda)$ are $\{4,-4\}$ and $\{2,-2\}$, so $s\left(e_{a}(\lambda)\right)=2$.

Let $X$ be the set of all infinite sequences $\mathbf{v}=\left(v_{1}, v_{2}\right.$, $\left.\ldots, v_{k}, \ldots\right)$ such that $v_{i} \in \mathbb{R}$. Define $W$ to be the reflection group on $X$ generated by the reflections $(i, j)$ and $(i, j)_{-}(i, j \in$ $\left.\mathbb{Z}^{>0}\right)$, where

$$
\begin{aligned}
& (i, j)\left(v_{1}, \ldots, v_{i}, \ldots, v_{j}, \ldots\right)=\left(v_{1}, \ldots, v_{j}, \ldots, v_{i}, \ldots\right), \\
& (i, j)_{-}\left(v_{1}, \ldots, v_{i}, \ldots, v_{j}, \ldots\right) \\
& \quad=\left(v_{1}, \ldots,-v_{j}, \ldots,-v_{i}, \ldots\right) .
\end{aligned}
$$

Rui and Si classified the blocks of BMW algebras with $o(q)>$ $n$ [13]. Based on this result, it was shown in [6] that two simple $\mathscr{B}_{n}(r, q)$-modules $D^{f, \lambda}$ and $D^{\ell, \mu}$ are in the same block if and only if $e_{a}(\lambda) \in W e_{a}(\mu)$. For $(f, \lambda) \in \Lambda_{n}$, let $\mathbf{B}_{n}(\lambda)$ be the block containing $\lambda$. Now we define $\mathbf{B}(\lambda)$ to be the set of partitions in the $W$-orbit of $\lambda$. So we have $\mathbf{B}(\lambda)=\bigcup_{m} \mathbf{B}_{m}(\lambda)$ where the union is taken over all $m$ such that $(\ell, \lambda) \in \Lambda_{m}$ for some $\ell$.

We consider the projection functor $\operatorname{Proj}_{\lambda}$ from the category of $\mathscr{B}_{n}(r, q)$-module to the block of $\mathscr{B}_{n}(r, q)$ which contains $\Delta(f, \lambda)$. So we have

$$
\operatorname{res}_{n} D^{f, \lambda}=\bigoplus_{\mathbf{B}(\mu)} \operatorname{Proj}_{\mu} \circ \operatorname{res}_{n} D^{f, \lambda} .
$$

By Theorem 4, we know that the direct sum can be taken over all blocks $\mathbf{B}(\mu)$ with $\mu \in \operatorname{supp}(\lambda)$, where $\operatorname{supp}(\lambda)=\left\{\mu \in \Lambda^{+}\right.$| $(\ell, \mu) \rightarrow(f, \lambda)$, for some $\left.f, \ell \in \mathbb{Z}^{\geq 0}\right\}$.

According to the definition of $s(\lambda)$, it is easy to see that there are three cases to consider depending on the relation between $s(\lambda)$ and $s(\mu)$ with $\mu \in \operatorname{supp}(\lambda)$ :

(1) $s(\lambda)=s(\mu)$,

(2) $s(\lambda)=s(\mu)-1$,

(3) $s(\lambda)=s(\mu)+1$.

Now, we need some notation in order to state the result of case (1).
Definition 8 (see [15]). Two partitions $\lambda$ and $\mu$ are said to be translation equivalent if

(a) $\mathbf{B}(\lambda) \cap \operatorname{supp}(\mu)=\{\lambda\}$ and $\mathbf{B}(\mu) \cap \operatorname{supp}(\lambda)=\{\mu\}$;

(b) for each $\lambda^{\prime} \in \mathbf{B}(\lambda)$, there is unique $\mu^{\prime} \in \mathbf{B}(\mu)$ such that $\mathbf{B}(\lambda) \cap \operatorname{supp}\left(\mu^{\prime}\right)=\left\{\lambda^{\prime}\right\}$ and $\mathbf{B}(\mu) \cap \operatorname{supp}\left(\lambda^{\prime}\right)=\left\{\mu^{\prime}\right\}$.

Proposition 9 (see [6]). Let $(f, \lambda) \in \Lambda_{n},(\ell, \mu) \in \Lambda_{n-1}$, and $\mu \in \operatorname{supp}(\lambda)$. If $s(\lambda)=s(\mu)$, then $\lambda$ and $\mu$ are translation equivalent.

Theorem 10. Let $(f, \lambda) \in \Lambda_{n},(\ell, \mu) \in \Lambda_{n-1}$, and $\mu \in \operatorname{supp}(\lambda)$. If $s(\lambda)=s(\mu)$, then

$$
\operatorname{Proj}_{\mu} \circ \operatorname{res}_{n} D^{f, \lambda}=D^{\ell, \mu} .
$$

Proof. According to the theory of cellular algebra, we have an epimorphism $\psi: \Delta(f, \lambda) \rightarrow D^{f, \lambda}$. Applying the functor $\operatorname{Proj}_{\mu} \circ$ res $_{n}$ to $\psi$, we have an epimorphism $\Delta(\ell, \mu) \rightarrow \operatorname{Proj}_{\mu} \circ$ $\operatorname{res}_{n} D^{f, \lambda}$ by Theorem 4 and Proposition 9. Hence Proj ${ }_{\mu}$ 。 $\operatorname{res}_{n} D^{f, \lambda}$ has simple head $D^{\ell, \mu}$.

If $D^{k, \nu}$ is in the socle of $\operatorname{Proj}_{\mu} \circ \operatorname{res}_{n} D^{f, \lambda}$, then $D^{k, v}$ must be a composition factor of $\Delta(\ell, \mu)$. So, we have

$$
\begin{aligned}
0 & \neq \operatorname{Hom}\left(\Delta(k, v), \operatorname{Proj}_{\mu} \circ \operatorname{res}_{n} D^{f, \lambda}\right) \\
& \cong \operatorname{Hom}\left(\Delta(k, v), \operatorname{res}_{n} D^{f, \lambda}\right) \\
& \cong \operatorname{Hom}\left(\operatorname{ind}_{n-1} \Delta(k, v), D^{f, \lambda}\right) \\
& \cong \operatorname{Hom}\left(\operatorname{Proj}_{\lambda} \circ \operatorname{ind}_{n-1} \Delta(k, v), D^{f, \lambda}\right) \\
& =\operatorname{Hom}\left(\Delta\left(k^{\prime}, v^{\prime}\right), D^{f, \lambda}\right) .
\end{aligned}
$$

The last equality follows from Definition 8 and Proposition 9.

Since $\operatorname{Hom}\left(\Delta\left(k^{\prime}, v^{\prime}\right), D^{f, \lambda}\right) \neq 0$, we have $\nu^{\prime}=\lambda$. Hence we have $\nu=\mu$.

So $\operatorname{Proj}_{\mu} \circ \operatorname{res}_{n} D^{f, \lambda}$ has simple head $D^{\ell, \mu}$ and simple socle $D^{\ell, \mu}$. However, the composition factors of $\operatorname{Proj}_{\mu} \circ \operatorname{res}_{n} D^{f, \lambda}$ must be the composition factors of $\Delta(\ell, \mu)$ and $[\Delta(\ell, \mu)$ : $\left.D^{\ell, \mu}\right]=1$, so we have $\operatorname{Proj}_{\mu} \circ \operatorname{res}_{n} D^{f, \lambda}=D^{\ell, \mu}$.

In order to deal with case (2) and case (3), we need some notation here.

Definition 11 (see [15]). Suppose that $\lambda^{\prime} \in \operatorname{supp}(\lambda)$. We say $\lambda^{\prime}$ separates $\lambda^{-}$and $\lambda^{+}$if $\lambda^{-} \neq \lambda^{+}$with one of $\lambda^{+}$or $\lambda^{-}$being equal to $\lambda$ and

(1) $\lambda^{\prime}$ is the unique element of $\mathbf{B}\left(\lambda^{\prime}\right) \cap \operatorname{supp}\left(\lambda^{-}\right)$;

(2) $\lambda^{\prime}$ is the unique element of $\mathbf{B}\left(\lambda^{\prime}\right) \cap \operatorname{supp}\left(\lambda^{+}\right)$;

(3) $\lambda^{-}$and $\lambda^{+}$are the unique pair of elements of $\mathbf{B}\left(\lambda^{-}\right) \cap$ $\operatorname{supp}\left(\lambda^{\prime}\right)$.

For $\mathbf{p} \in \mathscr{R}(\lambda)$ (or $\mathscr{A}(\lambda)$ ), we denote the partition corresponding to the Young diagram $Y(\lambda) \backslash \mathbf{p}($ or $Y(\lambda) \cup \mathbf{p})$ by $\lambda-\mathbf{p}$ (or by $\lambda+\mathbf{p}$ ). 
Proposition 12. Let $(f, \lambda) \in \Lambda_{n},\left(\ell, \lambda^{\prime}\right) \in \Lambda_{n-1}$, and $\lambda^{\prime} \in$ $\operatorname{supp}(\lambda)$. If $s(\lambda)=s\left(\lambda^{\prime}\right)-1$, then $\lambda^{\prime}$ separates $\lambda$ and $\lambda-\mathbf{p}-\widetilde{\mathbf{p}}$ for some $\mathbf{p}, \widetilde{\mathbf{p}} \in \mathscr{R}(\lambda)$ or $\lambda^{\prime}$ separates $\lambda$ and $\lambda+\mathbf{p}^{\prime}+\widetilde{\mathbf{p}}^{\prime}$ for some $\mathbf{p}^{\prime}, \widetilde{\mathbf{p}}^{\prime} \in \mathscr{A}(\lambda)$.

Proof. The first statement is [6, Proposition 4.11]. For the second statement, it is just needed to replace $\lambda$ and $\lambda-\mathbf{p}-\widetilde{\mathbf{p}}$ by $\lambda+\mathbf{p}^{\prime}+\widetilde{\mathbf{p}}^{\prime}$ and $\lambda$ in the proof of [6, Proposition 4.11], respectively.

Remark 13. Fixing the above notation, we assume that $\lambda^{+}$J $\lambda^{-}$. So, we write $\lambda^{+}$and $\lambda^{-}$instead of $\lambda$ and $\lambda-\mathbf{p}-\widetilde{\mathbf{p}}$ and write $\lambda^{+}$and $\lambda^{-}$instead of $\lambda+\mathbf{p}^{\prime}+\widetilde{\mathbf{p}}^{\prime}$ and $\lambda$, respectively.

Theorem 14. Let $\left(f, \lambda^{+}\right),\left(f+1, \lambda^{-}\right) \in \Lambda_{n},\left(f, \lambda^{\prime}\right) \in \Lambda_{n-1}$, and $\lambda^{\prime} \in \operatorname{supp}\left(\lambda^{+}\right)$be as above. Then we have

(1) $\operatorname{Proj}_{\lambda^{\prime}} \circ \operatorname{res}_{n} D^{f, \lambda^{+}}=D^{f, \lambda^{\prime}}$,

(2) $\operatorname{Proj}_{\lambda^{\prime}} \circ \operatorname{res}_{n} D^{f+1, \lambda^{-}}=0$.

Proof. Similar to the proof of Theorem 10, we have two epimorphisms $\psi^{+}: \Delta\left(f, \lambda^{+}\right) \rightarrow D^{f, \lambda^{+}}$and $\psi^{-}: \Delta(f+$ $\left.1, \lambda^{-}\right) \rightarrow D^{f+1, \lambda^{-}}$. Applying the functor $\operatorname{Proj}_{\lambda^{\prime}} \circ$ res $_{n}$ to $\psi^{+}$ and $\psi^{-}$, we have two epimorphisms $\Delta\left(f, \lambda^{\prime}\right) \rightarrow \operatorname{Proj}_{\lambda^{\prime}}$ 。 $\operatorname{res}_{n} D^{f, \lambda^{+}}$and $\Delta\left(f, \lambda^{\prime}\right) \rightarrow \operatorname{Proj}_{\lambda^{\prime}} \circ \operatorname{res}_{n} D^{f+1, \lambda^{-}}$by Theorem 4 and Proposition 12. Hence $\operatorname{Proj}_{\lambda^{\prime}} \circ \operatorname{res}_{n} D^{f, \lambda^{+}}$and $\operatorname{Proj}_{\lambda^{\prime}} \circ$ $\operatorname{res}_{n} D^{f+1, \lambda^{-}}$are either 0 or have simple head $D^{\ell, \lambda^{\prime}}$.

By $[9$, Corollary 5.8] and Proposition 12, we have an exact sequence

$$
\begin{aligned}
0 & \longrightarrow \Delta\left(f+1, \lambda^{-}\right) \longrightarrow \operatorname{Proj}_{\lambda^{+}} \circ \operatorname{ind}_{n-1} \Delta\left(f, \lambda^{\prime}\right) \\
& \longrightarrow \Delta\left(f, \lambda^{+}\right) \longrightarrow 0 .
\end{aligned}
$$

So, we have

$$
\begin{aligned}
& \operatorname{Hom}\left(\Delta\left(f, \lambda^{\prime}\right), \operatorname{Proj}_{\lambda^{\prime}} \circ \operatorname{res}_{n} D^{f, \lambda^{+}}\right) \\
& \cong \operatorname{Hom}\left(\Delta\left(f, \lambda^{\prime}\right), \operatorname{res}_{n} D^{f, \lambda^{+}}\right) \\
& \cong \operatorname{Hom}\left(\operatorname{ind}_{n-1} \Delta\left(f, \lambda^{\prime}\right), D^{f, \lambda^{+}}\right) \\
& \cong \operatorname{Hom}\left(\operatorname{Proj}_{\lambda^{+}} \circ \operatorname{ind}_{n-1} \Delta\left(f, \lambda^{\prime}\right), D^{f, \lambda^{+}}\right) \neq 0 .
\end{aligned}
$$

Hence $\operatorname{Proj}_{\lambda^{\prime}} \circ \operatorname{res}_{n} D^{f, \lambda^{+}}$has simple head $D^{f, \lambda^{\prime}}$.

With the same reason as Theorem 10, we complete the proof of (1).

Since $\left[\Delta\left(f, \lambda^{\prime}\right): D^{f, \lambda^{\prime}}\right]=1$, according to the proof of (1), the unique copy of $D^{f, \lambda^{\prime}}$ must come from $\operatorname{Proj}_{\lambda^{\prime}} \circ \operatorname{res}_{n} D^{f, \lambda^{+}}$. So $\operatorname{Proj}_{\lambda^{\prime}} \circ \operatorname{res}_{n} D^{f+1, \lambda^{-}}$cannot have simple head $D^{f, \lambda^{\prime}}$; this implies that $\operatorname{Proj}_{\lambda^{\prime}} \circ \operatorname{res}_{n} D^{f+1, \lambda^{-}}=0$.

Keeping the same notation, for case (3), we need to describe $\operatorname{Proj}_{\lambda^{+}} \circ \operatorname{res}_{n} D^{f, \lambda^{\prime}}$ for $\left(f, \lambda^{\prime}\right) \in \Lambda_{n}$ and $\left(f-1, \lambda^{+}\right)$, $\left(f, \lambda^{-}\right) \in \Lambda_{n-1}$. In this paper, we only describe the head and socle of $\operatorname{Proj}_{\lambda^{+}} \circ \operatorname{res}_{n} D^{f, \lambda^{\prime}}$.
Theorem 15. Let $\left(f, \lambda^{\prime}\right) \in \Lambda_{n}$ and $\left(f-1, \lambda^{+}\right),\left(f, \lambda^{-}\right) \in \Lambda_{n-1}$ be as above. Then

(1) if $f=0$, then $\operatorname{Proj}_{\lambda^{+}} \circ \operatorname{res}_{n} D^{0, \lambda^{\prime}}=D^{0, \lambda^{-}}$;

(2) if $f>0$, then $\operatorname{Proj}_{\lambda^{+}} \circ \operatorname{res}_{n} D^{f, \lambda^{\prime}}$ has simple head $D^{f-1, \lambda^{+}}$and simple socle $D^{f-1, \lambda^{+}}$.

Proof. When $f=0, \Delta\left(0, \lambda^{\prime}\right)$ can be considered as the cell module of Hecke algebra $\mathscr{H}_{n}$. However, under our assumption, Hecke algebra $\mathscr{H}_{n}$ is semisimple. So we have $D^{0, \lambda^{\prime}}=$ $\Delta\left(0, \lambda^{\prime}\right)$. Similarly, we have $D^{0, \lambda^{-}}=\Delta\left(0, \lambda^{-}\right)$. So, (1) follows from Theorem 4 and Proposition 12. phism

Similar to the proof of Theorem 10, we have an epimor-

$$
\phi: \operatorname{Proj}_{\lambda^{+}} \circ \operatorname{res}_{n} \Delta\left(f, \lambda^{\prime}\right) \longrightarrow \operatorname{Proj}_{\lambda^{+}} \circ \operatorname{res}_{n} D^{f, \lambda^{\prime}} .
$$

If $D^{\ell, \mu}$ is in the head of $\operatorname{Proj}_{\lambda^{+}} \circ \operatorname{res}_{n} D^{f, \lambda^{\prime}}$, it must be in the head of $\operatorname{Proj}_{\lambda^{+}} \circ \operatorname{res}_{n} \Delta\left(f, \lambda^{\prime}\right)$.

By $[9$, Corollary 5.8] and Proposition 12, we have an exact sequence

$$
\begin{aligned}
0 \longrightarrow \Delta\left(f, \lambda^{-}\right) \longrightarrow \operatorname{Proj}_{\lambda^{+}} \circ \operatorname{res}_{n} \Delta\left(f, \lambda^{\prime}\right) \\
\longrightarrow \Delta\left(f-1, \lambda^{+}\right) \longrightarrow 0 .
\end{aligned}
$$

It is easy to see that $D^{f-1, \lambda^{+}}$is in the head of $\operatorname{Proj}_{\lambda^{+}}$。 $\operatorname{res}_{n} \Delta\left(f, \lambda^{\prime}\right)$. Note that

$$
\operatorname{Proj}_{\lambda^{+}} \circ \operatorname{res}_{n} \Delta\left(f, \lambda^{\prime}\right)=\operatorname{Proj}_{\lambda^{+}} \circ \operatorname{ind}_{n-2} \Delta\left(f-1, \lambda^{\prime}\right) .
$$

So, we have

$$
\begin{aligned}
& \operatorname{Hom}\left(\operatorname{Proj}_{\lambda^{+}} \circ \operatorname{res}_{n} \Delta\left(f, \lambda^{\prime}\right), D^{f, \lambda^{-}}\right) \\
& \cong \operatorname{Hom}\left(\operatorname{Proj}_{\lambda^{+}} \circ \operatorname{ind}_{n-2} \Delta\left(f-1, \lambda^{\prime}\right), D^{f, \lambda^{-}}\right) \\
& \cong \operatorname{Hom}\left(\Delta\left(f-1, \lambda^{\prime}\right), \operatorname{Proj}_{\lambda^{\prime}} \circ \operatorname{res}_{n-1} D^{f, \lambda^{-}}\right)=0 .
\end{aligned}
$$

The last equality follows from Theorem 14(2).

Hence $\operatorname{Proj}_{\lambda^{+}} \circ \operatorname{res}_{n} \Delta\left(f, \lambda^{\prime}\right)$ has simple head $D^{f-1, \lambda^{+}}$. It follows that $\operatorname{Proj}_{\lambda^{+}} \circ \operatorname{res}_{n} D^{f, \lambda^{\prime}}$ has simple head $D^{f-1, \lambda^{+}}$.

Assume that $D^{\ell, \mu}$ is in the socle of $\operatorname{Proj}_{\lambda^{+}}{ }^{\circ} \operatorname{res}_{n} D^{f, \lambda^{\prime}}$. Then

$$
\begin{aligned}
0 & \neq \operatorname{Hom}\left(\Delta(\ell, \mu), \operatorname{Proj}_{\lambda^{+}} \circ \operatorname{res}_{n} D^{f, \lambda^{\prime}}\right) \\
& \cong \operatorname{Hom}\left(\Delta(\ell, \mu), \operatorname{res}_{n} D^{f, \lambda^{\prime}}\right) \\
& \cong \operatorname{Hom}\left(\operatorname{ind}_{n-1} \Delta(\ell, \mu), D^{f, \lambda^{\prime}}\right) \\
& \cong \operatorname{Hom}\left(\operatorname{Proj}_{\lambda^{\prime}} \circ \operatorname{ind}_{n-1} \Delta(\ell, \mu), D^{f, \lambda^{\prime}}\right) .
\end{aligned}
$$

Hence the socle of $\operatorname{Proj}_{\lambda^{+}} \circ \operatorname{res}_{n} D^{f, \lambda^{\prime}}$ consists of $D^{f-1, \lambda^{+}}$ and $D^{f, \lambda^{-}}$by Proposition 12 . 
Suppose that $D^{f, \lambda^{-}}$is in the socle of $\operatorname{Proj}_{\lambda^{+}} \circ \operatorname{res}_{n} D^{f, \lambda^{\prime}}$. Consider the set $\widetilde{\Lambda_{n-1}}=\left\{(\ell, \mu) \in \Lambda_{n-1} \mid D^{\ell, \mu}\right.$ is a composition factor of $\left.\operatorname{Proj}_{\lambda^{+}} \circ \operatorname{res}_{n} D^{f, \lambda^{\prime}}\right\}$. According to the assumption, $\left(f, \lambda^{-}\right) \in \widetilde{\Lambda_{n-1}}$ and must be the maximal one in the poset $\widehat{\Lambda_{n-1}}$; hence $D^{f, \lambda^{-}}$must come from $\Delta\left(f, \lambda^{-}\right)$. So, we have $\phi\left(\Delta\left(f, \lambda^{-}\right)\right) \supseteq D^{f, \lambda^{-}}$.

Hence we have the following commutative diagram:

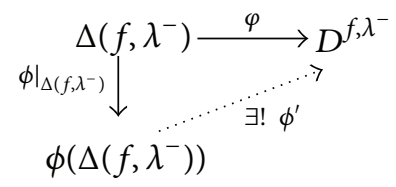

It follows that $\phi\left(\Delta\left(f, \lambda^{-}\right)\right)=\operatorname{ker} \phi^{\prime} \oplus D^{f, \lambda^{-}}$. This implies that $\phi\left(\Delta\left(f, \lambda^{-}\right)\right)=D^{f, \lambda^{-}}$. With the exact sequence (12), we have the following commutative diagram:

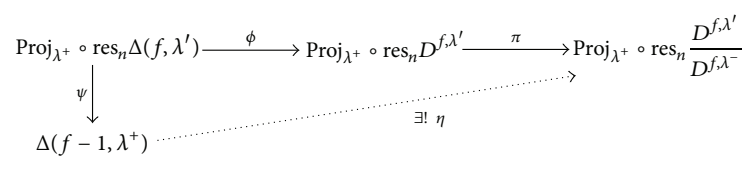

Since $\phi$ and $\pi$ are two epimorphisms, $\eta$ is an epimorphism. This means that the composition factors of $\operatorname{Proj}_{\lambda^{+}}$。 $\operatorname{res}_{n} D^{f, \lambda^{\prime}}$ must be composition factors of $\Delta\left(f-1, \lambda^{+}\right)$except one copy of $D^{f, \lambda^{-}}$.

However, we have

$$
\begin{aligned}
& {\left[\operatorname{Proj}_{\lambda^{+}} \circ \operatorname{res}_{n} D^{f, \lambda^{\prime}}: D^{f-1, \lambda^{+}}\right]} \\
& \quad=\operatorname{dim} \operatorname{Hom}\left(P\left(f-1, \lambda^{+}\right), \operatorname{Proj}_{\lambda^{+}} \circ \operatorname{res}_{n} D^{f, \lambda^{\prime}}\right) \\
& \quad \cong \operatorname{dim} \operatorname{Hom}\left(\operatorname{Proj}_{\lambda^{\prime}} \circ \operatorname{ind}_{n-1} P\left(f-1, \lambda^{+}\right), D^{f, \lambda^{\prime}}\right) \\
& \quad=2 .
\end{aligned}
$$

The last equality follows from the proof of [6, Proposition 4.13].

It is in contradiction with $\left[\Delta\left(f-1, \lambda^{+}\right): D^{f-1, \lambda^{+}}\right]=1$. Hence $D^{f, \lambda^{-}}$cannot be in the socle of $\operatorname{Proj}_{\lambda^{+}} \circ \operatorname{res}_{n} D^{f, \lambda^{\prime}}$. It follows that $D^{f-1, \lambda^{+}}$is in the socle of $\operatorname{Proj}_{\lambda^{+}} \circ \operatorname{res}_{n} D^{f, \lambda^{\prime}}$. Since we have proved that $\operatorname{Proj}_{\lambda^{+}} \operatorname{ores}_{n} D^{f, \lambda^{\prime}}$ has simple head $D^{f-1, \lambda^{+}}$, $\operatorname{Proj}_{\lambda^{+}} \circ \operatorname{res}_{n} D^{f, \lambda^{\prime}}$ has simple socle $D^{f-1, \lambda^{+}}$.

\section{Conflict of Interests}

The author declares that there is no conflict of interests regarding the publication of this paper.

\section{References}

[1] R. Brauer, "On algebras which are connected with the semisimple continuous groups," Annals of Mathematics, vol. 38, no. 4, pp. 857-872, 1937.
[2] J. S. Birman and H. Wenzl, "Braids, link polynomials and a new algebra," Transactions of the American Mathematical Society, vol. 313, no. 1, pp. 249-273, 1989.

[3] J. Murakami, "The Kauffman polynomial of links and representation theory," Osaka Journal of Mathematics, vol. 24, no. 4, pp. 745-758, 1987.

[4] A. N. Kirillov and Y. N. Reshetihikhin, "Representations of the algebras $U_{q}(s l(2)), q$-orthogonal polynomials and invariants of links," in Infinite-Dimensional Lie Algebras and Groups, V. G. Kac, Ed., pp. 285-339, World Scientific, Singapore, 1989.

[5] M. De Visscher and P. Martin, "On Brauer algebra simple modules over the complex field," Transactions of the American Mathematical Society, 2012.

[6] X. Xu, "Decomposition numbers of cyclotomic NW and BMW algebras," Journal of Pure and Applied Algebra, vol. 217, no. 6, pp. 1037-1053, 2013.

[7] H. R. Morton, "A basis for the Birman-Wenzl algebra," http://arxiv.org/abs/1012.3116.

[8] J. J. Graham and G. I. Lehrer, "Cellular algebras," Inventiones Mathematicae, vol. 123, no. 1, pp. 1-34, 1996.

[9] J. Enyang, "Specht modules and semisimplicity criteria for Brauer and BIRman-Murakami-Wenzl algebras," Journal of Algebraic Combinatorics, vol. 26, no. 3, pp. 291-341, 2007.

[10] H. Rui and M. Si, "Gram determinants and semisimplicity criteria for Birman-Wenzl algebras," Journal Für die Reine und Angewandte Mathematik, vol. 2009, no. 631, pp. 153-179, 2009.

[11] C. C. Xi, "On the quasi-heredity of Birman-Wenzl algebras," Advances in Mathematics, vol. 154, no. 2, pp. 280-298, 2000.

[12] J. A. Green, M. Schocker, and K. Erdmann, Polynomial Representations of $\mathrm{GL}_{n}$, vol. 830 of Lecture Notes in Mathematics, Springer, Berlin, Germany, 2nd edition, 2007.

[13] H. Rui and M. Si, "Blocks of Birman-Murakami-Wenzl algebras," International Mathematics Research Notices, no. 2, pp. 452-486, 2011.

[14] H. Rui and M. Si, "The representations of cyclotomic BMW algebras, II," Algebras and Representation Theory, vol. 15, no. 3, pp. 551-579, 2012.

[15] A. Cox, M. De Visscher, and P. Martin, "Alcove geometry and a translation principle for the Brauer algebra," Journal of Pure and Applied Algebra, vol. 215, no. 4, pp. 335-367, 2011. 


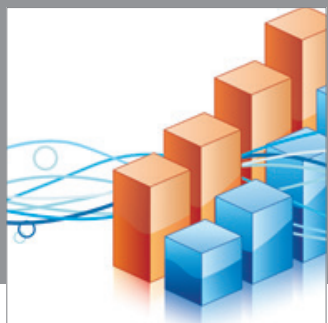

Advances in

Operations Research

mansans

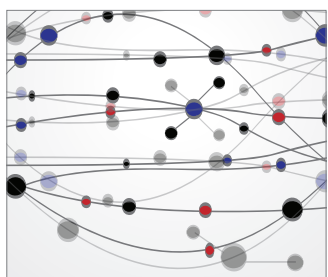

The Scientific World Journal
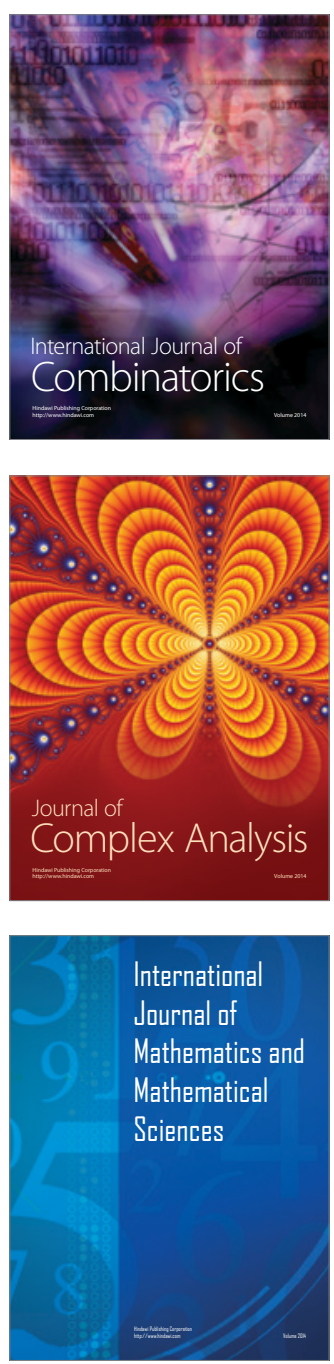
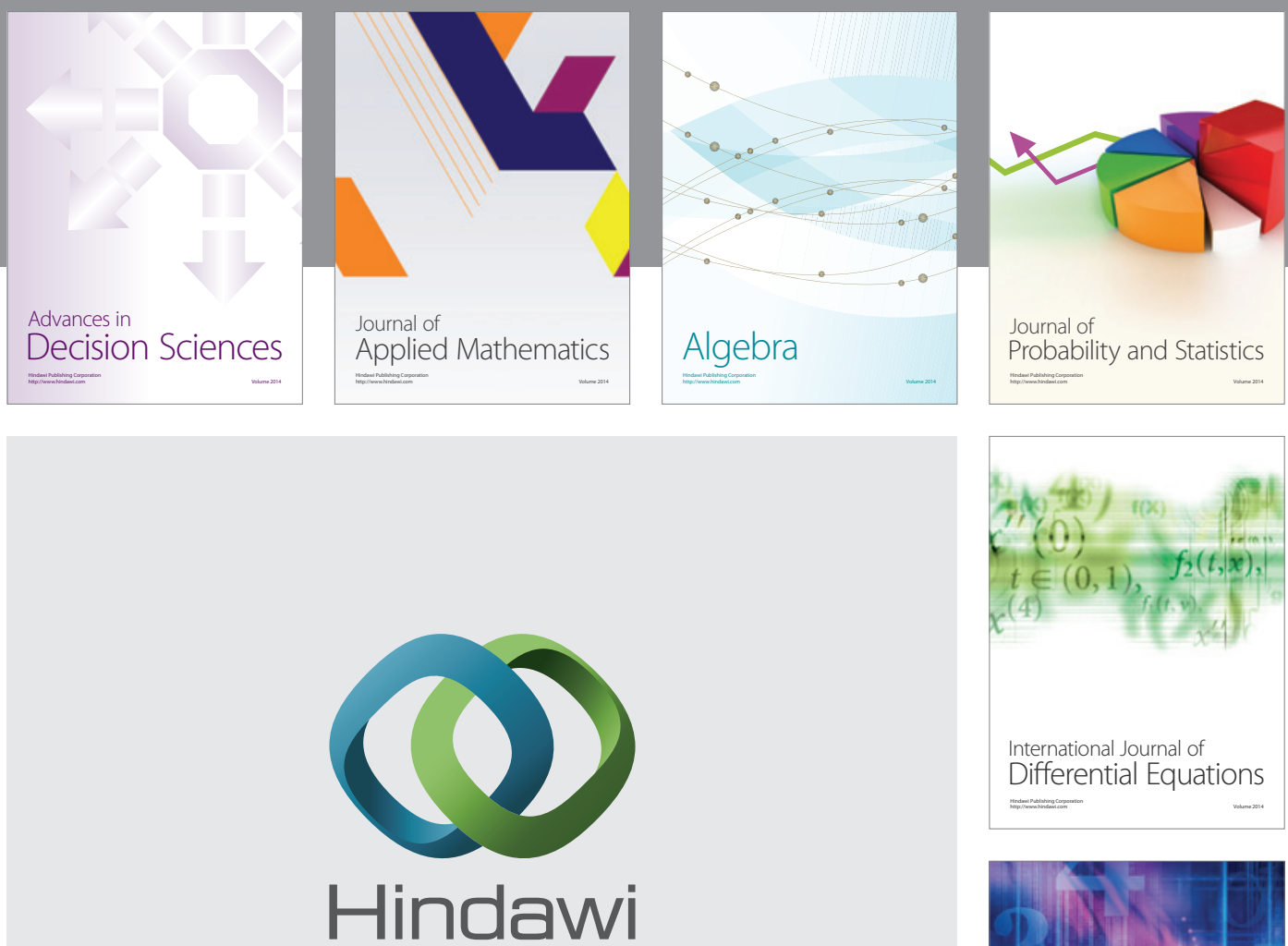

Submit your manuscripts at http://www.hindawi.com
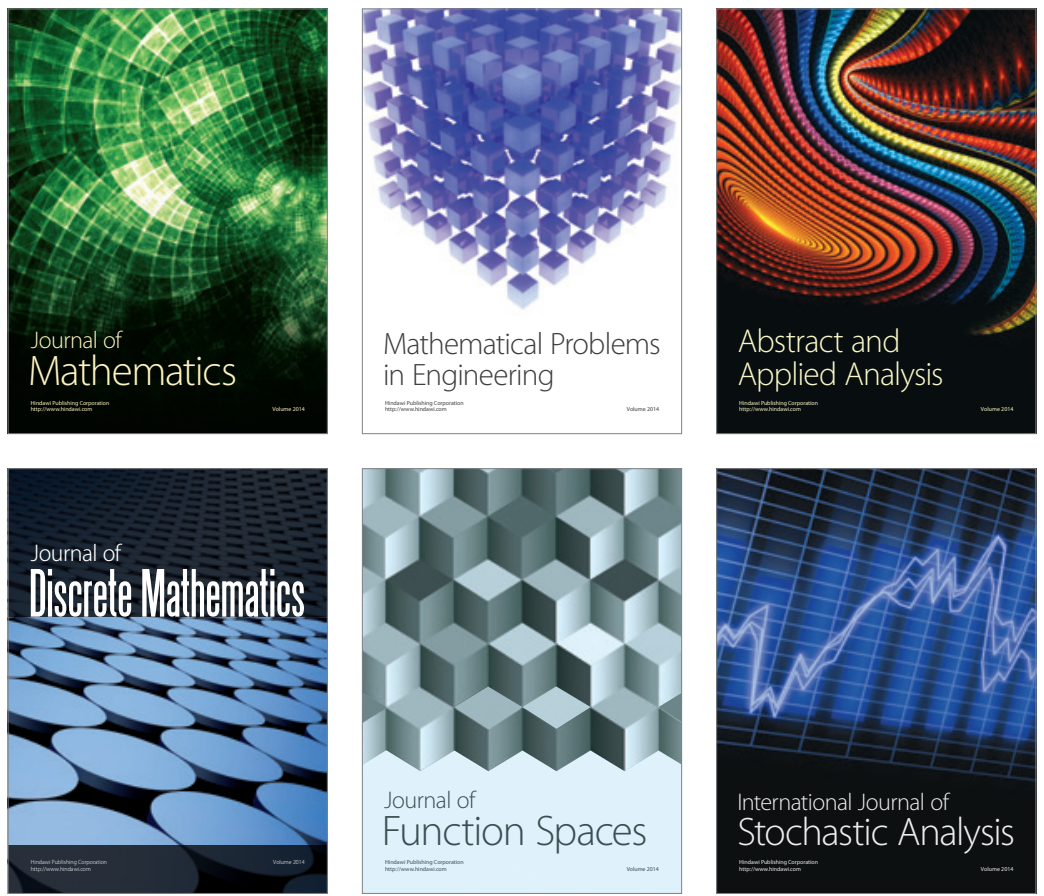

Journal of

Function Spaces

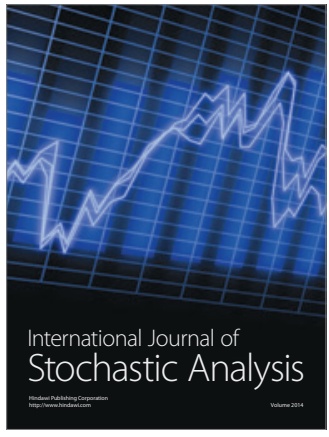

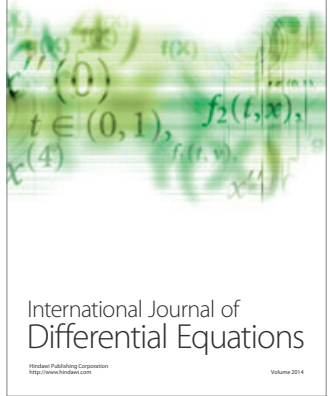
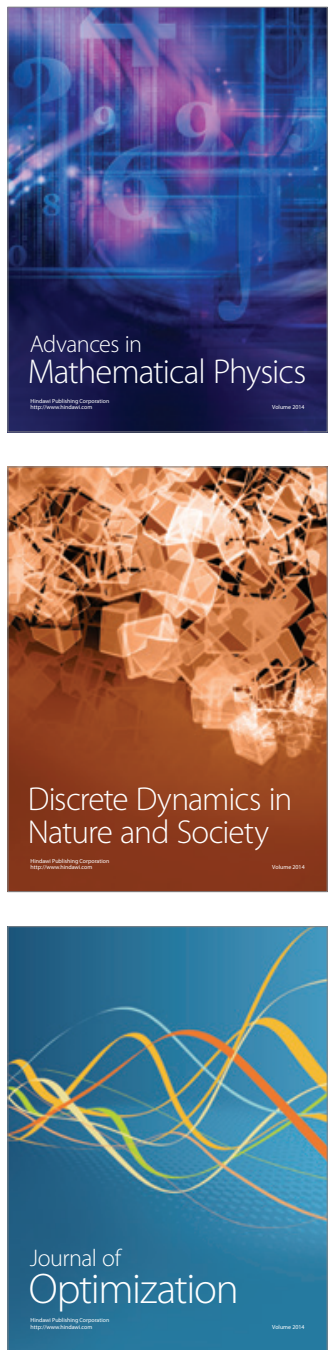